\title{
Sensitivity and Dynamic Range Considerations for Homodyne Detection Systems
}

\author{
Jaggard, Dwight L.; King, Ray J
}

Published in:

I E E E Transactions on Instrumentation and Measurement

Link to article, DOI:

10.1109/TIM.1973.4314183

Publication date:

1973

Document Version

Publisher's PDF, also known as Version of record

Link back to DTU Orbit

Citation (APA):

Jaggard, D. L., \& King, R. J. (1973). Sensitivity and Dynamic Range Considerations for Homodyne Detection Systems. I E E E Transactions on Instrumentation and Measurement, 22(4), 331-338.

https://doi.org/10.1109/TIM.1973.4314183

\section{General rights}

Copyright and moral rights for the publications made accessible in the public portal are retained by the authors and/or other copyright owners and it is a condition of accessing publications that users recognise and abide by the legal requirements associated with these rights.

- Users may download and print one copy of any publication from the public portal for the purpose of private study or research.

- You may not further distribute the material or use it for any profit-making activity or commercial gain

- You may freely distribute the URL identifying the publication in the public portal 


\title{
Sensitivity and Dynamic Range Considerations for Homodyne Detection Systems
}

\author{
DWIGHT L. JAGGARD, STUDENT MEMBER, IEEE, AND RAY J. KING, MEMBER, IEEE
}

\begin{abstract}
The effects of modulation frequency, RF reference power, and external bias upon the sensitivity and dynamic range of microwave homodyne detection systems was measured for point contact diodes and low $1 / f$ noise Schottky and backward diodes. The measurements were made at $4.89 \mathrm{GHz}$ using a signal to noise ratio of $3 \mathrm{~dB}$ and a detection system bandwidth of $10 \mathrm{~Hz}$. Maximum sensitivities of -135 , -150 , and $-145 \mathrm{dBm}$, and dynamic ranges of 92,110 , and $124 \mathrm{~dB}$ were measured for the point contact, Schottky, and backward diodes at modulation frequencies of 30,30 , and $3 \mathrm{kHz}$, respectively. It was found that the level of RF reference signal needed to obtain the maximum sensitivity was equal to or somewhat above the point where the diode changes from square law to linear detection. The results are significant in that previously reported homodyne sensitivities (not necessarily maximum) were on the order of -90 to $-130 \mathrm{dBm}$ for point contact diodes and no data are available for Schottky and backward diodes. Significantly improved stability, sensitivity, and dynamic range can be achieved using these low $1 / f$ noise devices, the correct external bias, and the optimum RF reference power.
\end{abstract}

\section{INTRODUCTION}

$\mathrm{H}$ OMODYNE (synchronous) detection is a process whereby a modulated signal which is to be detected is mixed with a CW reference signal of the same frequency in a nonlinear device, and the output signal is bandpass filtered. The detection process is basically the same as heterodyne detection except that the intermediate frequency (IF) is zero, and the detected signal generally lies in the audio range. Lowfrequency detector noise therefore plays a dominant role and conventional methods of specifying mixer diode characteristics are usually not very useful.

Although comparisons of the operational characteristics of various devices for video and heterodyne detection have appeared [1]-[5], little attention has been given to the operational characteristics of these devices when used as homodyne detectors. Notable exceptions are the papers by Brodwin et al. [6], who studied the sensitivity characteristics of point contact detectors as a function of reference signal amplitude, and by

Manuscript received May 1, 1973; revised August 7, 1973. This work was supported by the Wisconsin Alumni Research Foundation and the National Science Foundation under Grant GK-21218. This paper was presented at the 1973 Electrical and Electronic Measurement and Test Instrument Conference (EEMTIC), Ottawa, Ont., Canada, May 15-17.

D. L. Jaggard was with the Department of Electrical Engineering, University of Wisconsin, Madison, Wis. 53706. He is now with the Division of Engineering and Applied Science, Steel Laboratory, California Institute of Technology, Pasadena, Calif. 91109.

R. J. King is with the Laboratory of Electromagnetic Theory, Technical University of Denmark, Lyngby, Denmark, on leave from the Department of Electrical Engineering, University of Wisconsin, Madison, Wis. 53706.
Mathers [7], Richmond [8], and O'Brien [9] who have given typical sensitivities which were obtained using either point contact diodes or barretters. These early papers gave little attention to such questions as the behavior of diode $1 / f$ or "flicker" noise at low frequencies and the effect of externally applied bias when detecting in the homodyne mode. Also, Schottky and backward diodes are two additional very useful low noise detecting devices, and no evaluation of their homodyne detection characteristics has been made. To satisfy this need we have attempted to establish criteria for evaluating these three different diode types by investigating the quantitative effects of externally applied bias, modulation frequency, and reference signal level upon the sensitivity and dynamic range of homodyne detection systems. These parameters were studied using a radio frequency of $4.89 \mathrm{GHz}$. Although the results depend to some degree upon the radio frequency, the particular diode, its mount, and associated bias and output circuitry, they are generally adequate to show how the sensitivity and dynamic range of the homodyne detector can be maximized.

The results show maximum sensitivities $S_{m}$ on the order of $-135,-150$, and $-145 \mathrm{dBm}$ and maximum dynamic ranges $D_{m}$ of 92,110 , and $125 \mathrm{~dB}$ for the point contact, Schottky, and backward diodes at modulation frequencies of 30,30 , and $3 \mathrm{kHz}$, respectively, using a bandwidth of $10 \mathrm{~Hz}$. The sensitivity values for the point contact diode are 5 to $45 \mathrm{~dB}$ better than previously reported results [6]-[9].

\section{THEORY}

Consider two signals of the same radian frequency $\omega$ where

$$
\begin{aligned}
& V_{r}=A e^{j \omega t} \\
& V_{i}=b\left(1+m \cos \omega_{m} t\right) e^{j(\omega t+\phi)} .
\end{aligned}
$$

The CW reference signal $V_{r}$ is of amplitude $A$, the information signal $V_{i}$ is of amplitude $b$ and is double-sideband with carrier (DSBWC) amplitude modulated with modulation index $m$. The radian modulation frequency is $\omega_{m}\left(\omega_{m} \ll \omega\right)$, and $V_{i}$ differs in phase from $V_{r}$ by phase angle $\phi$. Using the phasor diagram shown in Fig. 1, and noting that at low levels the detector has a square-law response, the output is proportional to $E_{t}^{2}\left(=\left(V_{r}+V_{i}\right)^{2}\right.$. Thus using the law of cosines and retaining only those terms at $\omega_{m}$, the detected output is

$$
V_{0}=K_{l} A b m\left(\frac{b}{A}+\cos \phi\right) \cos \omega_{m} t
$$




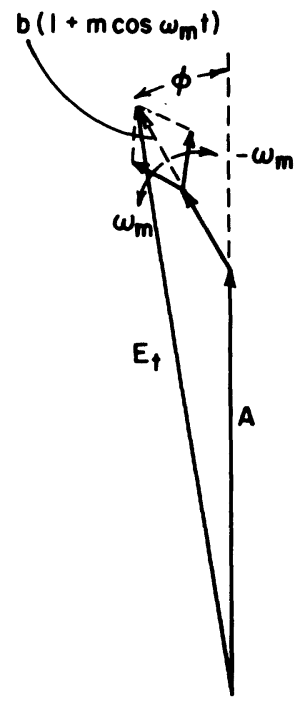

Fig. 1. Phasor diagram showing addition of reference signal $A$ and DSBWC amplitude modulated information signal to yield total signal $E_{t}$ arriving at detector.

where $K_{l}$ is the low level gain constant. The $b / A$ term in (3) represents the effect of the unsuppressed carrier in the information signal which is given by the first term in (2), and contributes to both phase [10]-[12] and amplitude measurement errors [11], [13], [14]. Its presence is most objectionable when making phase measurements, wherein $\phi$ is adjusted in one of the channels by using a phase shifter so that (3) is nulled. The phase error can be made negligible small by requiring $b<<A$, e.g., for $b / A<-30 \mathrm{~dB}$ the phase error is less $1.8^{\circ}$. Thus the amount of phase error which can be tolerated determines the lower level of $A$ which in turn determines the upper bound on the dynamic range of $b$. In the following we shall assume that $b / A<-30 \mathrm{~dB}$, and so the first term in (3) can be ignored. Alternatively, by using a balanced modulator and/or mixer, a multiplying suppression factor will be introduced to reduce this error term [11], [12]. Similar errors using single sideband modulation have also been analyzed [11].

At higher levels of $A$, the detector characteristic becomes linear and simply detects the envelope of the total input $E_{t}$. For $b<<A$, the output portion of $E_{t}$ at $\omega_{m}$ is

$$
V_{0}=K_{h} b m \cos \phi \cos \omega_{m} t
$$

where $K_{h}$ is the high level gain constant. The important difference between (3) and (4) is that the output for linear detection is not a function of the reference signal amplitude $A$ whereas for square-law detection (3) shows that the output is a linear function of $A$ [6], [11]. In either case, the output is directly proportional to $b \cos \phi$ (assuming $b<<A$ ) and can therefore be used to make phase and amplitude measurements on the information channel signal arriving at the detector. Here we are only concerned with the dynamic range of $b$ and the ability of the system to measure small values of $b$, i.e., sensitivity, and so we shall always set $\cos \phi=1$.

Aside from the errors in phase and amplitude introduced by not having $b<<A$, a second type of measurement error can occur due to unwanted signals, e.g., leakage or reflection of a portion of the modulated information signal into the reference channel or vice versa. These errors can be minimized by using isolators, circulators, well matched magic tees, directional couplers, attenuators, and tuners.

The effect of the reference channel signal level $A$, the detector noise and externally applied bias, and the modulation frequency $\omega_{m}$, are all important in homodyne detection. $A$ should be large since a large dynamic range of $b$ is desired, and since $b<<A$ must hold for error free phase measurements. But very large values can damage the diode, cause large losses due to extreme impedance mismatch, or cause the rectified signal current to develop a reverse self-bias through the dc load resistance. Another disadvantage arises from the increased low-frequency noise generated within the detector. Since the output becomes independent of $A$ at high levels, a point is reached beyond which further increases in $A$ simply increase the conversion loss or add noise with a corresponding reduction in the output signal to noise ratio. On the other hand, when $A$ is decreased into the square-law region, the detected signal output decreases in direct proportion to $A$. Since the detector noise is at its lowest value and essentially constant, the net result is again a reduction in the output signal to noise ratio. An optimum value of $A$ can therefore be found near or somewhat above the transition region from square-law to linear detection which will give the maximum sensitivity $S_{m}$. This point will be dependent upon diode type and mount, external bias and bias circuit impedance, and modulation frequency. Furthermore, it will be necessary to determine the tradeoffs which can be made to yield the greatest dynamic range in $b$.

Most readers are familiar with the characteristics of point contact diodes when used as mixers [15], [16] and so the mixing characteristics of the Schottky and backward diodes will be briefly compared with this device. More comprehensive comparisons are available [1] , [3] , [4]. First the advantages: because construction of the Schottky and backward diodes does not involve a metal whisker-semiconductor junction, they are inherently more stable with temperature and mechanical vi. brations, have a higher burnout rating, and can be operated at higher local oscillator power levels (A for homodyne applica. tions) without significantly increasing the diode noise. Still another advantage is that Schottky and backward diodes generate significantly lower noise (10 to $30 \mathrm{~dB})$ at low IF (IF is zero for homodyne). The point contact diode has a $1 / f$ noise power spectrum below the noise corner which lies between $100 \mathrm{kHz}$ and $1 \mathrm{MHz}$. Above the noise corner the noise is almost entirely shot and thermal which are constant for frequencies up to $10^{8}$ and $10^{13} \mathrm{~Hz}$, respectively, and in the range the noise from the Schottky and backward diodes is comparable to the point contact [1], [4], [17]. More importantly for homodyne applications, the Schottky diode also has a $1 / f$ noise spectrum but has a considerably lower noise corner which varies from $50 \mathrm{~Hz}$ to $30 \mathrm{kHz}$, while the backward diode noise generally increases more slowly than $1 / f$ as the frequency is reduced, and the noise corner can be as low as $1 \mathrm{kHz}$.

In the disadvantages column, the Schottky diode requires a small amount of external bias to conduct at low local oscillator power levels $(A)$. Otherwise the Schottky and point 
contact diodes have comparable IF impedances (100 to 500 $\Omega)$ and in many cases the Schottky can be used as a direct replacement for the point contact. However, special detector design considerations are required for the backward diode which is usually operated with zero bias and a very low impedance dc load. Furthermore, the IF impedance is quite low (40 to $100 \Omega$ ), and so a special IF amplifier is usually needed, e.g., transformer [4] or low impedance transistor amplifier [5].

Rough calculations using well-known formulas for receiver sensitivity give maximum sensitivity values of $-127,-149$, and $-153 \mathrm{dBm}$ for the point contact, Schottky, and backward diodes, respectively, at a modulation frequency of $1 \mathrm{kHz}$. These values compare favorably with the experimental results.

\section{THE EXPERIMENT}

Fig. 2 shows the $C$-band ( 3.95 to $5.85 \mathrm{GHz}$ ) microwave homodyne detection system that was used in the following experiments. The $\mathrm{CW}$ signal from the $1.5-\mathrm{W}$ klystron was divided into the reference and information channels by magic tee No. 1 which in conjunction with tuner $T_{1}$, isolators, and attenuators in each channel, provided a high degree of isolation between channels. The reference channel provided a reference signal $V_{r}$ of amplitude $A$, while the information channel provided a DSBWC amplitude modulated $(m=0.6)$ signal $V_{i}$ of amplitude $b$ (see (1) and (2)). These signals were combined by magic tee No. 2, which, in conjunction with tuner $T_{2}$ provided additional isolation between channels. The variable attenuators set the power levels so that $b<<A$. The position of the slotted line probe determined the phase angle $\phi$ between $V_{r}$ and $V_{i}$, and was always adjusted so that $\cos \phi=1$. Since $A \gg b, V_{r}$ synchronously switches the detector diode, giving an output of the form of (3) or (4).

The detector mount was coaxial and tunable with a dc return. It was observed that the optimum tuning of the mount was not measureably changed for levels of $A$ below or somewhat above that which gave maximum sensitivity, and so the tuning was performed for $A \cong-10 \mathrm{dBm}$ and held fixed for each diode at each external bias setting. The bias circuit had an internal impedance of $100 \mathrm{k} \Omega$, capable of supplying up to $1 \mathrm{~mA}$ external bias. The chosen bias current remained constant until $A$ exceeded its optimum value by about 5-10 dB.

The medium impedance low-noise preamplifier provided a voltage gain of 100 and had an input resistance of $100 \mathrm{k} \Omega$. This preamplifier, model 148L by Ithaco, was used for the point contact and Schottky diodes and had a noise figure of $4 \mathrm{~dB}$ for a source resistance of $200 \Omega$ at $30 \mathrm{kHz}$. The addition of a resistive load in parallel with the preamplifier input only degraded the sensitivity and made the diode response more dependent upon the level of $A$. Therefore, no additional external shunting load was used for any of the diode types.

The low impedance low noise preamplifier which was used for the backward diode provided a voltage gain of 1000 and had a dc resistance of $2 \Omega$ due to the transformer coupled input. The preamplifier, model G1 by Ithaco, had a noise figure of $0.5 \mathrm{~dB}$ for a source resistance of $75 \Omega$ at $300 \mathrm{~Hz}$, and provided the proper resistive load and near zero bias.

A compensated thermister bridge and power meter were

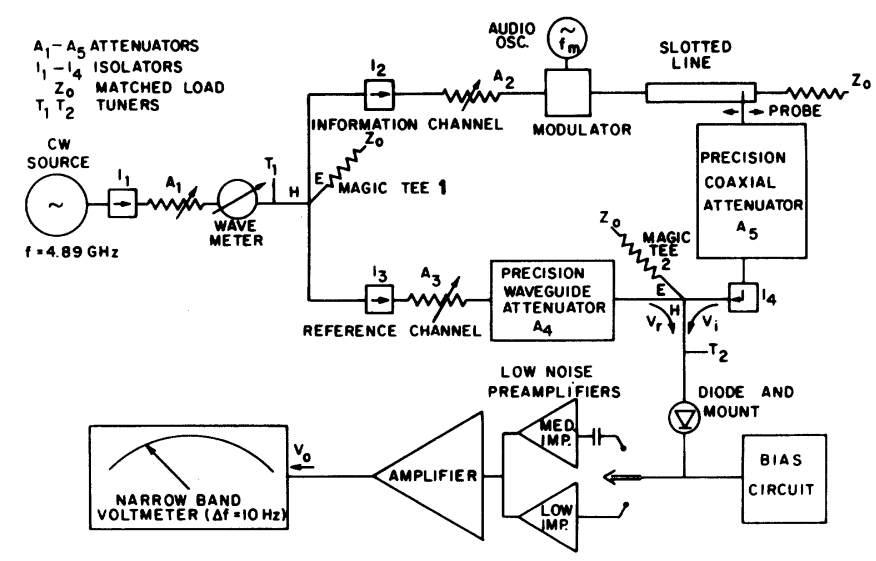

Fig. 2. Microwave homodyne system for testing detection characteristics of various diode types.

used to establish the power level of each channel at the detector mount and the precision attenuators were used to vary $A$ from +10 to $-40 \mathrm{dBm}$ and vary $b$ from -90 to $-140 \mathrm{dBm}$ for the point contact, or from -110 to $-160 \mathrm{dBm}$ for the more sensitive Schottky and backward diodes.

The first data taken from the point contact and Schottky diodes established the minimum detectable signal $b$ which was $3 \mathrm{~dB}$ above noise as a function of $A$ in the range $-40<A<$ $+10 \mathrm{~dB}$ using external bias as the parameter. This gave information needed to determine the optimum bias. The modulation frequency $f_{m}$ was held fixed at $1 \mathrm{kHz}$, and the bandwidth $\Delta f$ at $10 \mathrm{~Hz}$.

Next, data were taken to determine the output for fixed $b(=-70 \mathrm{dBm})$ versus $A$ using external bias as the parameter, and a fixed modulation frequency of $1 \mathrm{kHz}$. These results established the detector square law, transition, and linear response regions as a function of $A$ and external bias. These regions are defined according to whether the output slope was +1 for the square-law region or zero for the linear region, and the transition region was where the slope deviated more than $1 \mathrm{~dB}$ from these values. The results of this second part of the experiment were used to correlate the center of the transition region with $A_{0}$, the optimum value of $A$ which occurred at the point of maximum sensitivity as previously suggested in Section II.

In the third part of the experiment the minimum detectable signal $b$ was measured as a function of $A$ over the range $-40<$ $A<+10 \mathrm{dBm}$, using the modulation frequency between 100 $\mathrm{Hz}$ and $30 \mathrm{kHz}$ as a parameter and the optimum bias found from the first data. This established the maximum sensitivity $S_{m}$ and maximum dynamic range of $b, D_{m}$, as functions of $A$ and modulation frequency $f_{m}$.

In all of these experiments, errors due to attenuator settings and unsteady meter readings were of the order of $\pm 0.25 \mathrm{~dB}$ for $A$ and $\pm 1.0 \mathrm{~dB}$ for $b$.

\section{EXPERimental Results}

Two new notations will be useful in this and the following sections. For a given modulation frequency and external bias, $A_{d}$ refers to the value of $A$ where the maximum dynamic range $D_{m}$ occurs, while $A_{\mathrm{TR}}$ denotes the approximate center of the transition region. 


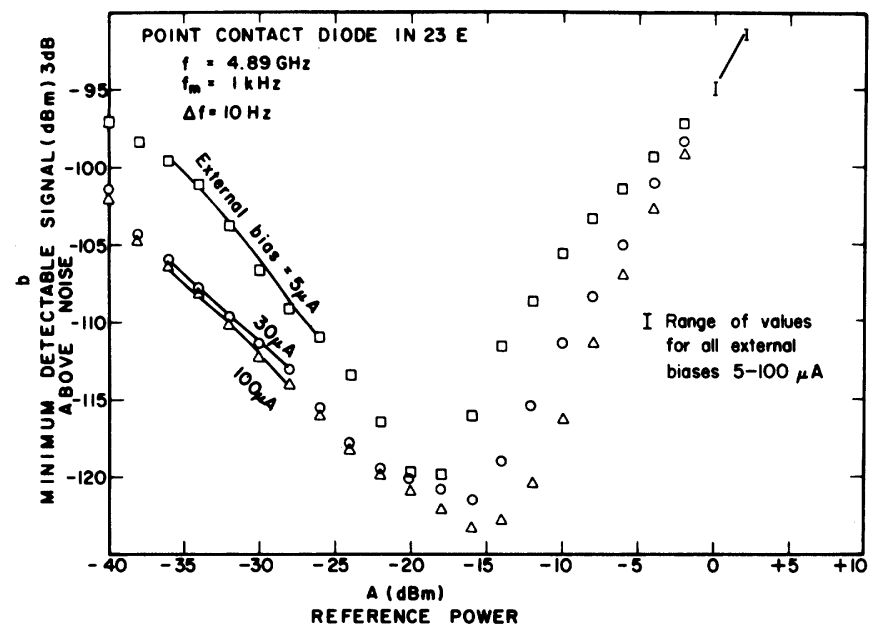

Fig. 3. Minimum detectable signal versus $A$ with external bias as parameter for point contact diode.

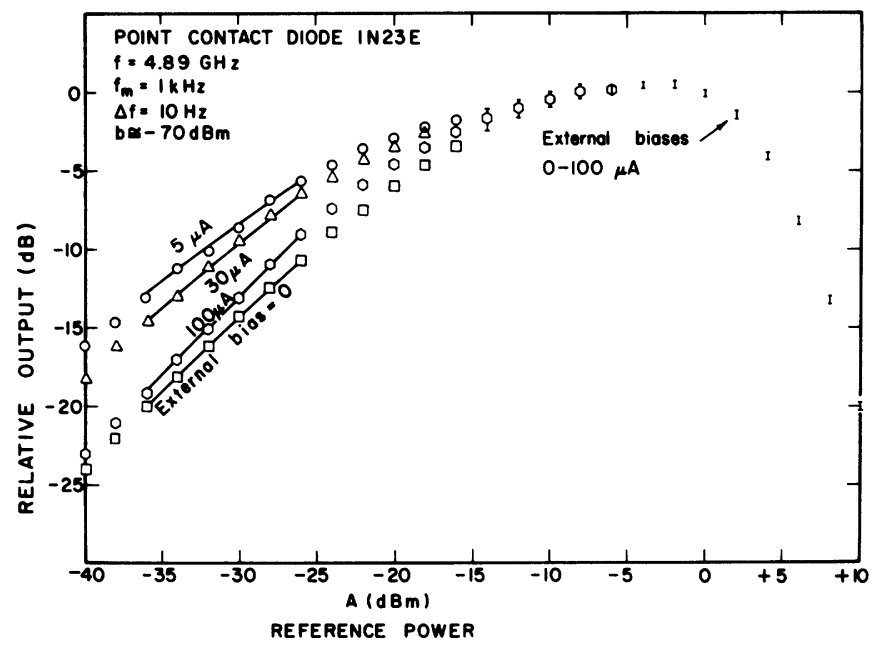

Fig. 4. Relative output of point contact diode versus $A$ with external bias as parameter and fixed information signal input $(-70 \mathrm{dBm})$.

\section{A. Point Contact Diode}

Figs. 3-5 show the results of the three experimental procedures outlined previously using the point contact diode. For $f_{m}=1 \mathrm{kHz}$, Fig. 3 shows the minimum detectable signal as a function of $A$ with external bias as a parameter. It is apparent that increasing the external bias increases both the maximum sensitivity and the dynamic range. It was observed that no improvement in maximum sensitivity could be realized by increasing the bias above $100 \mu \mathrm{A}$, and so curves for larger bias are not shown.

The upswing of the curves in Fig. 3 for $A>A_{0}$ is largely due to the onset of reverse self-bias which was caused by the flow of rectified RF current through the $100 \mathrm{k} \Omega$ internal resistance of the bias circuit. Of course, increased diode noise also plays a role. Consequently, if measures are taken to eliminate or compensate the reverse self-bias, these curves would display a somewhat broader minimum which would extend to higher values of $A$. This broadening would probably not improve the observed maximum sensitivity, but would increase the maximum dynamic range since $A$ could then be set somewhat above the optimum $A_{0}$ as measured from Fig. 3.

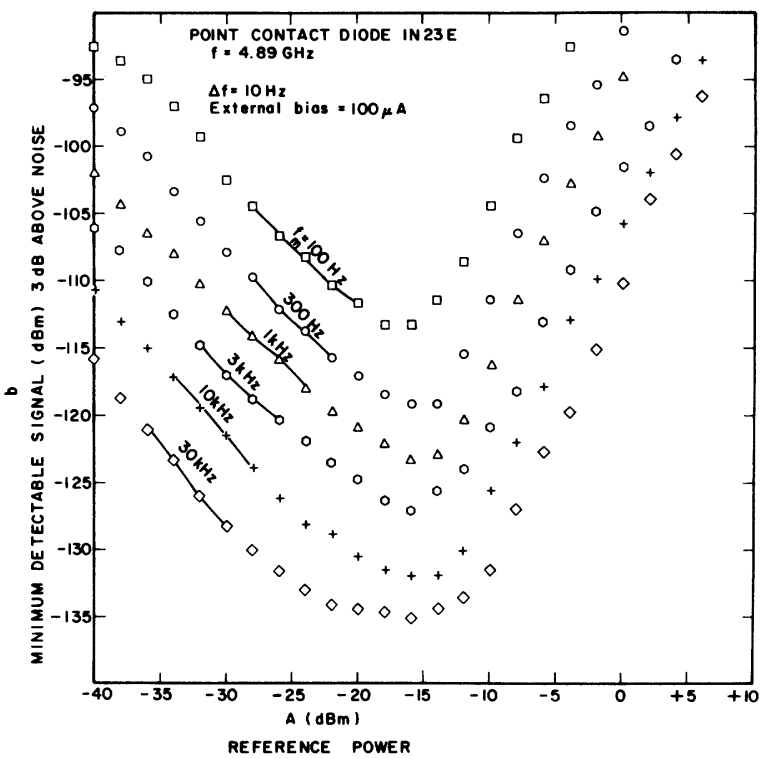

Fig. 5. Minimum detectable signal for point contact diode versus $A$ with fixed external bias and modulation frequency $f_{m}$ as parameter.

TABLE I

Maximum Sensitivity and Dynamic Range as Function of MOdUlation FreQuency For 1N23E CONTACT DIODE

\begin{tabular}{|c||c|c||c|c|}
\hline $\begin{array}{c}\mathrm{f}_{\mathrm{m}} \\
(\mathrm{Hz})\end{array}$ & $\begin{array}{c}\text { Maximum } \\
\text { Sensitivity } \\
\mathrm{s}_{\mathrm{m}}(\mathrm{dBm})\end{array}$ & $\begin{array}{c}\mathrm{A}_{\mathrm{o}} \\
(\mathrm{dBm})\end{array}$ & $\begin{array}{c}\text { Maximum Dynamic } \\
\text { Range } \\
\mathrm{D}_{\mathrm{m}}(\mathrm{dB})\end{array}$ & $\begin{array}{c}\mathrm{A}_{\mathrm{d}} \\
(\mathrm{dBm})\end{array}$ \\
\hline \hline 100 & -113 & -17 & 67 & -14 \\
300 & -119 & -16 & 74 & -14 \\
$1 \mathrm{k}$ & -123 & -16 & 79 & -14 \\
$3 \mathrm{k}$ & -127 & -16 & 82 & -13 \\
$10 \mathrm{k}$ & -132 & -16 & 88 & -12 \\
$30 \mathrm{k}$ & -135 & -16 & 92 & -11 \\
\hline
\end{tabular}

Unfortunately, this reverse self-bias effect was not noticed until shortly before the final paper was due for printing, and so it was not possible to repeat the experiments to observe the increased dynamic range which could be achieved. However, additional data are planned for a future note.

Fig. 4 shows the output of the diode as the level of $A$ is varied again using external bias as a parameter. For the $100-\mu \mathrm{A}$ curve, the detector is square-law for $A<-22 \mathrm{dBm}$, in the transition region for $-22 \mathrm{dBm}<A<-11 \mathrm{dBm}$ and linear for $-11 \mathrm{dBm}<A<+1 \mathrm{dBm}$. Note the broad transition region between the square-law and linear detection regions, especially for small bias. For $A<0 \mathrm{dBm}$ the reverse self-bias is not important here since the signal $b(=-70 \mathrm{dBm})$ is well above the noise level. However, its effect is quite pronounced for $A>0 \mathrm{dBm}$ where the sharp drop-off in detected output is observed.

For homodyne systems, operation is normally in the region where the curves in Fig. 4 begin to merge. Consequently, bias should be chosen on the basis of maximum sensitivity and not maximum output. According to Fig. 3, the optimum bias for the point contact diode was therefore taken as $100 \mu \mathrm{A}$ in Fig. 5 which shows the minimum detectable signal as a function of $A$ using $f_{m}$ as the parameter. Table I summarizes the results shown in Fig. 5.

In Table I, $A_{d}$ has been taken at the point where the slope 
begins to be greater than +1 and is therefore always somewhat greater than $A_{0}$. This is the point where any further increase in $A$ tends to reduce the minimum detectable signal (determining the low end of the dynamic range) at a rate which is greater than the increase at the upper end of the dynamic range, less $30 \mathrm{~dB}$ to assure that $b<<A$ as discussed in Section II. Symbolically,

$$
D_{m}=S_{d}+A_{d}-30 \mathrm{~dB}
$$

where $S_{d}$ is the magnitude of the sensitivity at $A=A_{d}$. As in the case of Fig. 3, $A_{d}$ would be increased if reverse self-bias does not occur, giving a corresponding increase in $D_{m}$.

The noise corner is reached above $f_{m}=30 \mathrm{kHz}$ since the curves of Fig. 5 are approximately equally spaced as the modulation frequency increases from $100 \mathrm{~Hz}$ to $30 \mathrm{kHz}$. $(30 \mathrm{kHz}$ was the largest modulation used since the frequency range of the wave analyzer was limited.) Theoretically, if only $1 / f$ noise is involved, the curves of Fig. 5 should be spaced $4.8 \mathrm{~dB}$ apart for frequency multiples of 3 , and spaced $5.2 \mathrm{~dB}$ apart for frequency multiples of 3.33. The slope of the curves in Fig. 5 for $A<A_{0}$ is approximately - 1, which would be expected if the only effect of increasing $A$ is to linearly increase the output [see (3)] without significantly increasing noise. The slope for $A \gg A_{0}$ is greater than +1 , which is largely due to the reverse self-bias effect already noted. Finally, $A_{0}$ appears very close to the center of the transition region $A_{\mathrm{TR}}$ as expected from the earlier discussion in Section II.

\section{B. Schottky Diode}

Following the same procedure as previously, the experimental results for the Schottky diode are shown in Figs. 6-8. Fig. 6 shows the minimum detectable signal as a function of $A$ with external bias as a parameter. As before, the curves merge for large $A$ showing that the effect of the external bias is insignificant compared to the rectified RF current due to the large reference signal $A$. Also note that the curves for different bias levels cross at low levels of $A$ suggesting that at these levels $(A<30 \mathrm{dBm})$ an optimum bias would be less than $100 \mu \mathrm{A}$. But this is not the usual laboratory situation since $A$ can generally be set in the transition or linear regions to increase the amplitude of the detected signal without significantly increasing the noise, and to increase the dynamic range. As in the case of the point contact, increasing the bias above $100 \mu \mathrm{A}$ gave no additional improvement in maximum sensitivity.

Fig. 7 clearly depicts a sharp transition between the squarelaw and linear regions for all values of external bias. For zero bias the Schottky barely conducts which accounts for the large slope of the zero bias curve, and adding a small amount of bias increases the output of the order of $20 \mathrm{~dB}$ for $A \cong-25 \mathrm{dBm}$. Another noteable feature of Fig. 7 is that the output is independent of $A$ over a range of about $25 \mathrm{~dB}$ when bias is present. Among other things, this would have application in automated amplitude measuring systems in which an electronic phase shifter is inserted in the reference branch to sweep the phase angle $\phi$ through a range of $180^{\circ}$. The output would vary as $b \cos \phi(t)$, and $b$ could be observed in real time. The unavoidable modulated insertion loss of the phase shifter

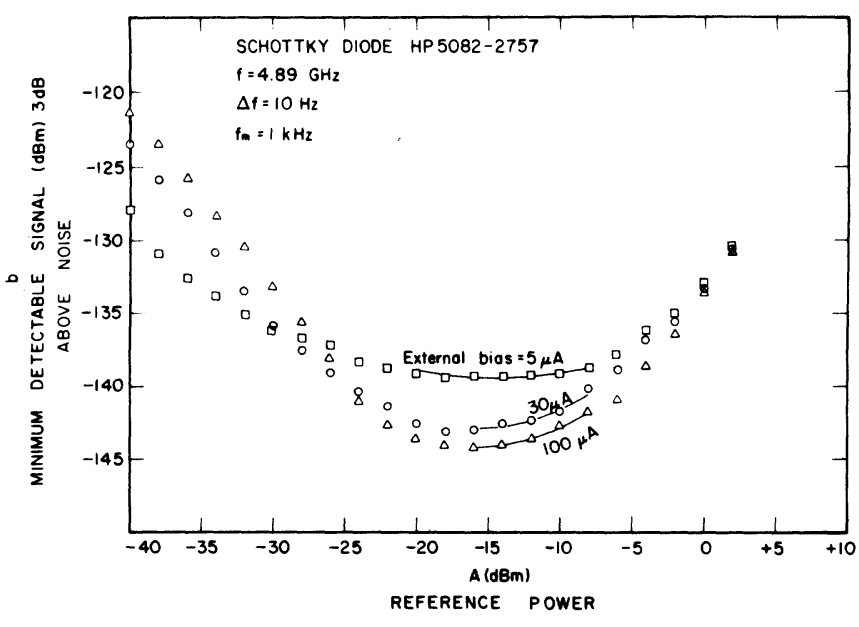

Fig. 6. Minimum detectable signal versus $A$ with external bias as parameter for Schottky diode.

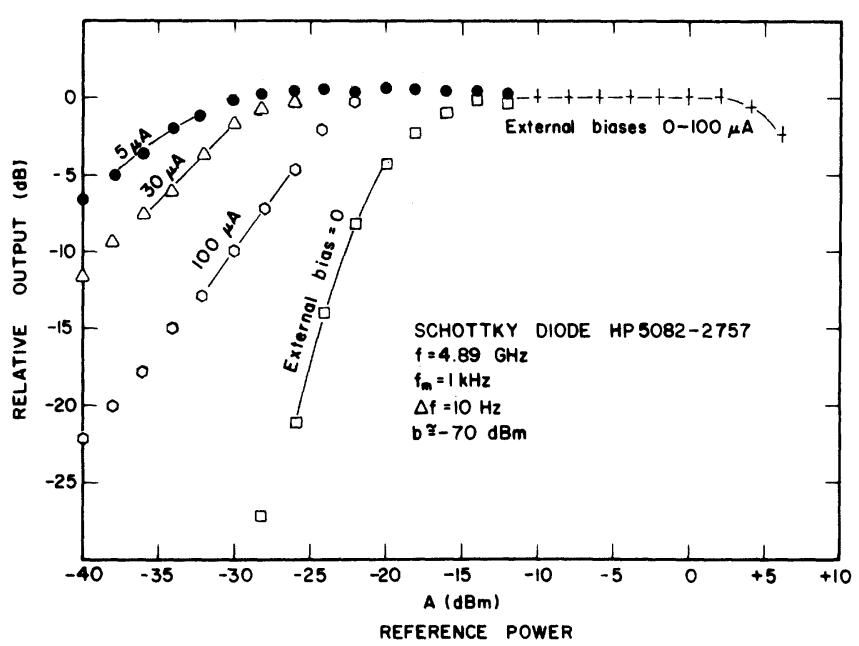

Fig. 7. Relative output of Schottky diode versus $A$ with external bias as parameter and fixed information signal input $(-70 \mathrm{dBm})$.

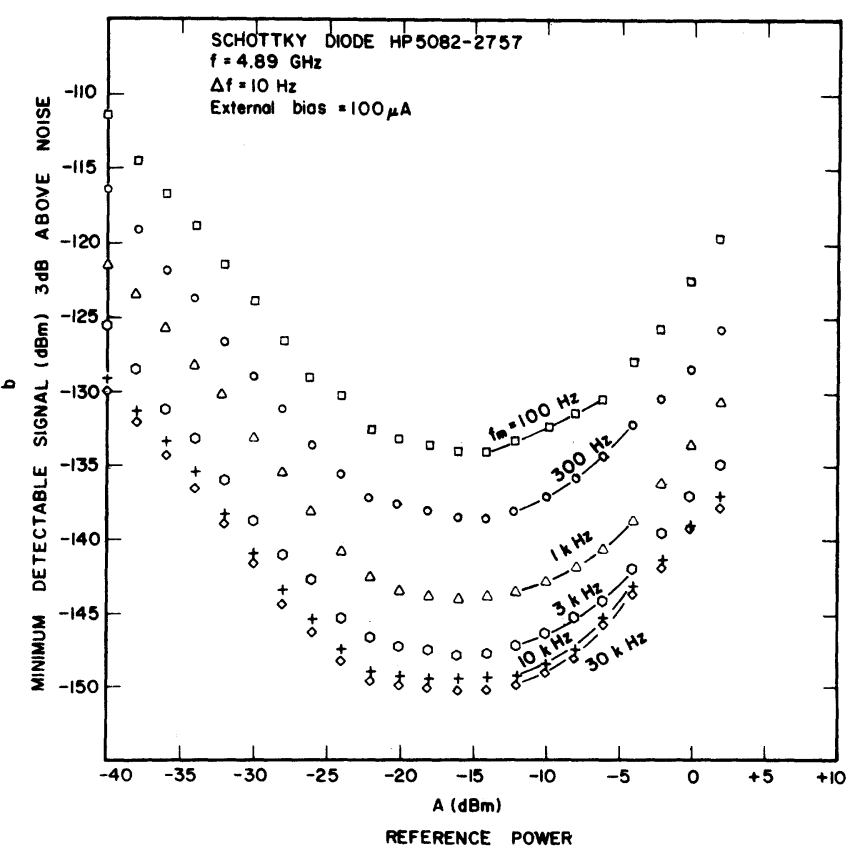

Fig. 8. Minimum detectable signal for Schottky diode versus $A$ with fixed external bias and modulation frequency $f_{m}$ as parameter. 
TABLE II

Maximum Sensitivity and Dynamic Range as Function of MODULATION FREQUENCY FOR HP5082-2757 SCHOTTKY DIODE

\begin{tabular}{|c||c|c|c|c|}
\hline $\begin{array}{c}\mathrm{f}_{\mathrm{m}} \\
(\mathrm{Hz})\end{array}$ & $\begin{array}{c}\text { Maximum } \\
\text { Sensitivity } \\
\mathrm{S}_{\mathrm{m}}(\mathrm{dBm})\end{array}$ & $\begin{array}{c}\mathrm{A}_{\mathrm{o}} \\
(\mathrm{dBm})\end{array}$ & $\begin{array}{c}\text { Maximum Dynamic } \\
\text { Range } \\
\mathrm{D}_{\mathrm{m}}(\mathrm{dB})\end{array}$ & $\begin{array}{c}\mathrm{A}_{\mathrm{d}} \\
(\mathrm{dBm})\end{array}$ \\
\hline 100 & -134 & -14 & 96 & -6 \\
300 & -138 & -14 & 98 & -6 to 0 \\
$1 \mathrm{k}$ & -144 & -16 & 106 & -6 to -2 \\
$3 \mathrm{k}$ & -148 & -16 & 108 & -6 to -2 \\
$10 \mathrm{k}$ & -149 & -14 to -18 & 109 & -8 to +2 \\
$30 \mathrm{k}$ & -150 & -14 to -18 & 110 & -8 to +2 \\
\hline
\end{tabular}

would then contribute insignificant error to the output signal amplitude. Finally, the sharp drop-off for $A>3 \mathrm{dBm}$ is due to the onset of severe reverse self-bias which also occurred for the point contact as shown in Fig. 4.

Using $100 \mu \mathrm{A}$ bias, Fig. 8 displays the behavior of the maximum sensitivity and dynamic range as a function of $A$ and the modulation frequency, and Table II summarizes these results.

Note that the noise corner of the Schottky diode is approached near $10 \mathrm{kHz}$ as evidenced by the bunching of the curves at the higher frequencies. For frequencies below $3 \mathrm{kHz}$ the curves are spread approximately 4.8 or $5.2 \mathrm{~dB}$ depending upon the frequency change, which is as expected from the influence of the $1 / f$ noise. For $A<A_{0}$, the slope is steeper than -1 which suggests that the conversion loss increases at low levels of $A$. At the higher levels, the slope is +1 near $A=0$ $\mathrm{dBm}$, which when compared with Fig. 5 for the point contact, indicates that the upper limit of the dynamic range for the Schottky is about $10 \mathrm{~dB}$ greater. In general, comparing Tables I and II, the sensitivity of the Schottky is 15 to $20 \mathrm{~dB}$ better than the point contact and the useable dynamic range is increased 20 to $30 \mathrm{~dB}$.

\section{Backward Diode}

Backward diodes normally operate with zero bias and a very low dc load, and so the only results graphically displayed here are those showing the minimum detectable signal versus $A$ with $f_{m}$ as a parameter (Fig. 9). The low source resistance of the backward diode required a special transformer input coupled preamplifier which only performed well for $f_{m}<3$ $\mathrm{kHz}$, but this was sufficiently high so that a good indication of the system performance could be established. Since the dc impedance of the transformer was only $2 \Omega$, reverse self-bias was no problem.

When the relative output was measured versus $A$ (not shown), it was found that the device is square law for $A<-6 \mathrm{dBm}$, and linear for $A>-2 \mathrm{dBm}$, with $A_{\mathrm{TR}} \simeq-4 \mathrm{dBm}$. Furthermore, the relative output was constant for $-2<A<+10 \mathrm{dBm}$. The experiment was not continued for still larger $A$ because of the danger of burning the diode out. These facts suggest that this diode has a very large dynamic range. Indeed, this can be seen by inspecting Fig. 9 and the summarizing data in Table III.

The most notable features of the data in Fig. 9 are that the point of maximum sensitivity $A_{0}$ is much larger than the corresponding points on Figs. 5 or 8 for the point contact or Schottky diodes, respectively. Another is that for $A<-15$

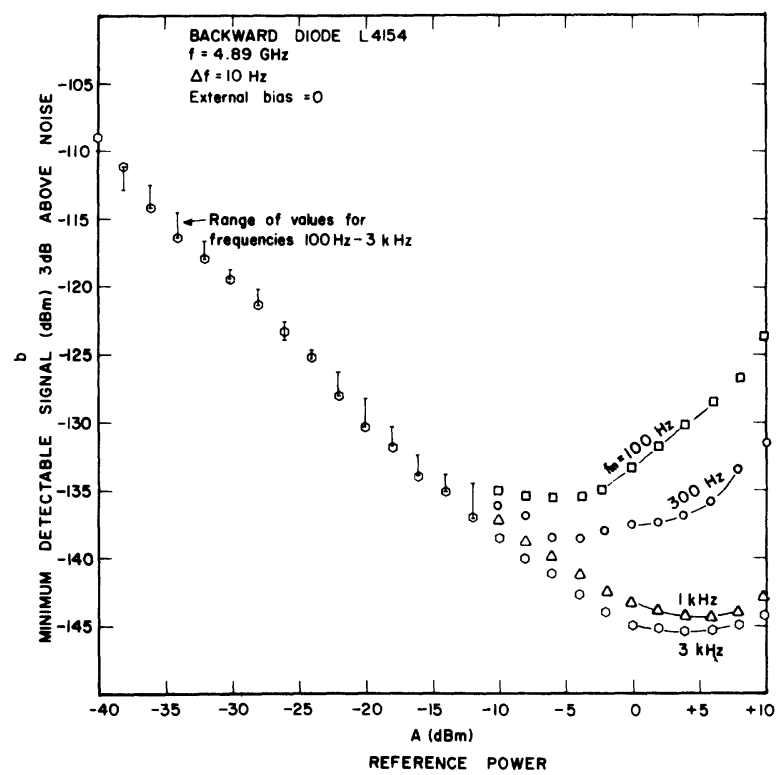

Fig. 9. Minimum detectable signal for backward diode versus $A$ with modulation frequency $f_{m}$ as parameter.

TABLE III

Maximum Sensitivity and Dynamic Range as Function of MODUlATION FREQUENCY FOR L4154 BACKWARD DIODE

\begin{tabular}{|c|c|c|c|c|}
\hline $\begin{array}{c}f_{m} \\
(\mathrm{~Hz})\end{array}$ & $\begin{array}{c}\text { Maximum } \\
\text { Sensitivity } \\
\mathrm{S}_{\mathrm{m}}(\mathrm{dBm})\end{array}$ & $\begin{array}{c}\mathrm{A}_{\mathrm{o}} \\
(\mathrm{dBm})\end{array}$ & $\begin{array}{c}\text { Maximum Dynamic } \\
\text { Range } \\
\mathrm{D}_{\mathrm{m}}(\mathrm{dBm})\end{array}$ & $\begin{array}{c}\mathrm{A}_{\mathrm{d}} \\
(\mathrm{dBm})\end{array}$ \\
\hline \hline 100 & -135 & -8 to -4 & 105 & +8 \\
300 & -138 & -6 to -4 & 112 & +6 to +10 \\
$1 \mathrm{k}$ & -144 & +4 to +6 & $\geq 122$ & $>+10$ \\
$3 \mathrm{k}$ & -145 & +2 to +6 & 2124 & $>+10$ \\
\hline
\end{tabular}

$\mathrm{dBm}$ there is little spread in the curves as would normally be expected from $1 / f$ noise. For $A>-15 \mathrm{dBm}$, the spread is quite nonuniform which suggests that the noise behaves as $1 / f^{n}$ where $n>1$. The bunching of the $1-\mathrm{kHz}$ and $3-\mathrm{kHz}$ curves for large $A$ could be due to an approaching noise corner frequency, or perhaps is due to the difference in frequency response of the transformer or different loading effects or a combination of these. Comparing Fig. 9 and Table III with Fig. 8 and Table II shows that the backward diode is capable of providing a dynamic range which is approximately $15 \mathrm{~dB}$ greater than that for the Schottky diode, and maximum sensitivities which are approximately equal for the two diodes.

A short final experiment confirmed the fact that the point contact and the Schottky diodes both have a $1 / f$ noise spectrum and noise corners greater than $30 \mathrm{kHz}$ and approximately $8 \mathrm{kHz}$, respectively. The backward diode, however, has a $1 / f^{1.5}$ noise spectrum, which was also shown by Eng [17], and a noise corner greater than $3 \mathrm{kHz}$.

\section{CONCLUSION}

Since these experiments only involved one diode of each type, generalizations to all other diodes of the same type should be made with some caution. However, trends and certain individual results should be noted.

1) By paying careful attention to the various parameters, optimum sensitivities which greatly exceed previously reported results are possible. Table IV compares these previous data to 
TABLE IV

Comparison of Homodyne SySTEm SENSITIVITIES ObTaINEd by VARIOUS AUTHORS REFERRED TO 10-Hz BANDWIDTH

\begin{tabular}{|c|c|c|c|c|c|c|}
\hline Author & $\underset{\mathrm{CW}}{\mathrm{CW}}$ & $\begin{array}{c}\text { Detector } \\
\text { Type }\end{array}$ & $\begin{array}{l}\text { Mod. Freq. } \\
\mathrm{f}_{\mathrm{m}} \text { in } \mathrm{Hz} \\
\text { (Mod. Type) }\end{array}$ & $\begin{array}{l}\text { Sensitivity, } \mathrm{S} \\
\text { or } \\
\text { (Max. } \begin{array}{l}\text { Sens, } \\
\mathrm{dBm}\end{array} \mathrm{s}_{\mathrm{m}} \text { ) }\end{array}$ & $\begin{array}{l}\text { Ref. Signal, A } \\
\text { or (0pt. Ref.) } \\
\text { Signal, A } \\
\quad \text { dBm }\end{array}$ & $\begin{array}{c}\text { Exter- } \\
\text { nal } \\
\text { Bias }\end{array}$ \\
\hline \multirow{3}{*}{$\begin{array}{l}\text { Brodwin, } \\
\text { et al., [6] }\end{array}$} & \multirow[b]{2}{*}{$9 \mathrm{GHz}$} & \multirow{2}{*}{$\begin{array}{l}\text { Point } \\
\text { Contact }\end{array}$} & 400 (DSBWC) & $(-97)$ & $(-20)$ & 0 \\
\hline & & & $2 \mathrm{k}(\mathrm{DSBWC})$ & $(-107)$ & $(-20)$ & 0 \\
\hline & $24 \mathrm{GHz}$ & $\begin{array}{c}\text { IN26 Point } \\
\text { Contact }\end{array}$ & ? (DSBWC) & $(-115)$ & $(-10)$ & 0 \\
\hline \multirow{2}{*}{ Mathers [7] } & \multirow{2}{*}{$\mathrm{X}$-b and } & $\begin{array}{l}\text { X421A } \\
\text { Point } \\
\text { Contact } \\
\end{array}$ & $1 \mathrm{k}(\mathrm{SSBSC})$ & $(-105)$ & $(-15)$ & 0 \\
\hline & & Barretter & $1 \mathrm{k}(\mathrm{SSBSC})$ & -100 & 0 & 0 \\
\hline \multirow{2}{*}{ Richmond [8] } & \multirow{2}{*}{$\mathrm{X}$-band } & $\begin{array}{c}\text { IN23 Point } \\
\text { Contact }\end{array}$ & $1 \mathrm{k}(\mathrm{DSBSC})$ & $(-89)$ & $(-6)$ & 0 \\
\hline & & $\begin{array}{c}821 \text { Sperry } \\
\text { Barretter }\end{array}$ & $1 \mathrm{k}$ (DSBSC) & -91 & +10 & $+5 \mathrm{MA}$ \\
\hline O'Brien [9] & $10 \mathrm{GHz}$ & $\begin{array}{l}\text { Point } \\
\text { Contact }\end{array}$ & $\begin{array}{l}100 \mathrm{k} \\
\text { (DSBSC) }\end{array}$ & -130 & $\approx-3$ & 0 \\
\hline \multirow{3}{*}{$\begin{array}{c}\text { Jaggard \& } \\
\text { King }\end{array}$} & \multirow{3}{*}{$4.89 \mathrm{GHz}$} & $\begin{array}{l}\text { IN23E } \\
\text { Point } \\
\text { Contact } \\
\text { (table 1) } \\
\end{array}$ & 30k (DSBWC) & $(-135)$ & $(-16)$ & $100 \mu \mathrm{A}$ \\
\hline & & $\begin{array}{l}\text { HP 5082-2757 } \\
\text { Schottky } \\
\text { (table 2) }\end{array}$ & 30k (DSBWC) & $(-150)$ & $(-18)$ to $(-14)$ & $100 \mu \mathrm{A}$ \\
\hline & & $\begin{array}{l}\text { L4154 } \\
\text { Backward } \\
\text { (table } 3 \text { ) }\end{array}$ & 3k（DSBWC） & $(-145)$ & $(+2)$ to $(+6)$ & 0 \\
\hline
\end{tabular}

the data obtained here. In the Brodwin et al. [6]data, the bandwidth was assumed to be $30 \mathrm{~Hz}$ since this information was not explicitly given. In all cases, the data are referred to a $10-\mathrm{Hz}$ noise bandwidth.

2) Very large dynamic ranges not available with other conventional detection techniques were also observed. Values of $D_{m}$ were measured as $92 \mathrm{~dB}$ for the point contact at $f_{m}=30$ $\mathrm{kHz}$ and $100-\mu \mathrm{A}$ bias (Table I), $110 \mathrm{~dB}$ for the Schottky at $f_{m}=30 \mathrm{kHz}$ and $100-\mu \mathrm{A}$ bias (Table II), and $124 \mathrm{~dB}$ for the backward diode at $f_{m}=3 \mathrm{kHz}$ and zero bias (Table III).

3) The optimum reference signal $A_{0}$ for point contacts lies in the transition region from square-law to linear detection, while $A_{0}$ lies 5-10 dB above this region for Schottky and backward diodes.

4) Below $50 \mathrm{GHz}$, the Schottky diode is almost an exact replacement for the point contact diode. At modulation frequencies below $10 \mathrm{kHz}$ the Schottky diode can improve homodyne sensitivity by 15 to $20 \mathrm{~dB}$ and increase the dynamic range by 20 to $30 \mathrm{~dB}$ when compared with the point contact diode (compare Tables I and II). The maximum sensitivities achieved for the point contact and Schottky diodes at $1 \mathrm{kHz}$ compare well with the predicted sensitivities of -127 and $-149 \mathrm{dBm}$, respectively.

If operation is well into the linear region, say $A>-15 \mathrm{dBm}$ there is little need for an external bias for either type (see Figs. 4 and 7). Adding a small amount of bias improves the maximum sensitivity slightly as seen from Figs. 3 and 6 .

5) The backward diode offers an excellent dynamic range because $A_{0}$ is quite large. For $f_{m}=1 \mathrm{kHz}, D_{m}$ is $43 \mathrm{~dB}$ greater than that of the point contact and the maximum sensitivity of the same order as that for the Schottky (compare Tables I-III). For the system used here, its low IF impedance probably prevented attainment of better sensitivities. Nevertheless, the maximum sensitivity of $-144 \mathrm{dBm}$ at $1 \mathrm{kHz}$ compares with the predicted sensitivity of $-153 \mathrm{dBm}$.

6) The point contact and Schottky diodes both have a clearly defined $1 / f$ noise spectrum at low frequencies. The backward diode has a $1 / f^{1.5}$ noise spectrum for low frequencies.

For sufficiently narrow bandwidths, homodyne systems operating with audio frequency modulation offer sensitivities which are equal to or greater than those of typical high IF hetrodyne systems which use much wider bandwidths.

Even greater dynamic ranges are possible for the point contact and Schottky diodes if the reverse self-bias effect is reduced or eliminated. Furthermore, $D_{m}$ can also be significantly increased for all diode types if a balanced modulator or detector is used, allowing removal of the restriction that $b / A<$ $-30 \mathrm{~dB}$ previously imposed. Perhaps other devices such as the space-charge-limited diode or the hot carrier thermoelectric detector have properties suitable for homodyne systems. These devices have excellent tangential sensitivities and no $1 / f$ noise [2].

\section{ACKNOWLEDGMENT}

The authors wish to thank Hewlett-Packard for donating four Schottky diodes (HP5082-2757), Philco-Ford for donating two backward diodes (L4154), and for the loan of a model 353 amplifier, and model 148L and G1 preamplifiers made by Ithaco from Mauer Company, Inc., Evanston, Ill.

\section{REFERENCES}

[1] R. E. Bayliss, E. Cabrera, and S. E. Howe, "Why a Schottkybarrier? Why a point-contact?," Microwaves, vol. 6, pp. 34-45, Mar. 1968.

[2] A. M. Cowley and H. O. Sorensen, "Quantitative comparison of solid-state microwaves detectors," IEEE Trans. Microwave Theory Tech., vol. MTT-14, pp. 588-602, Dec. 1966. 
[3] B. S. Siegal, "Schottky diodes-where we stand today," Microwaves, vol. 10, pp. 44-46, Apr. 1971.

[4] R. O. Wright, "The backward diode-when and how to use it," Microwaves, vol. 3, pp. 22-27, Dec. 1964.

[5] -, "New twist for backward diode help from low-noise amplifier," Electronics, vol. 39, pp. 74-77, July 11, 1966.

[6] M. E. Brodwin, C. M. Johnson, and W. M. Waters, "Low level synchronous mixing," in IRE Nat. Conv. Rec., vol. 1, pt. 10, pp. 52-57, Mar. 1953.

[7] G. W. C. Mathers, "Homodyne generator and detection system," in IRE Wescon Conv. Rec., vol. 1, pt. 1, pp. 194-200, 1957.

[8] J. H. Richmond, "Measurement of time-quadrature components of microwave signals," IRE Trans. Microwave Theory Tech., vol. MTT-3, pp. 13-15, Apr. 1955.

[9] B. B. O'Brien, "A pseudo-superheterodyne receiver for measuring phase and amplitude," IEEE Trans. Instrum. Meas., vol. IM-16, pp. 124-128, June 1967.

[10] G. E. Schafer, "A modulated subcarrier technique of measuring microwave phase shifts," IRE Trans. Instrum., vol. I-9, pp. $217-$ 219 , Sept. 1960.

[11] R. J. King, "Real time measurement of microwave parameters and EM fields," IEEE Trans. Instrum. Meas., vol. IM-21, pp. 2-10, Feb. 1972.

[12] B. A. Howarth and T. J. F. Pavlásek, "Analysis of automatic homodyne method amplitude and phase measurements," IEEE Trans. Microwave Theory Tech., vol. MTT-20, pp. 623-626, Sept. 1972.

[13] G. E. Schafer and R. R. Bowman, "A modulated sub-carrier technique of measuring microwave attenuation," Proc. Inst. Elec. Eng., vol. 109B(23), pp. 783-786, May 1962.

[14] W. E. Little, "Further analysis of the modulated subcarrier technique of attenuation measurement," IEEE Trans. Instrum. Meas., vol. IM-13, pp. 71-76, June-Sept. 1964.

[15] H. C. Torrey and C. A. Whitmer, Crystal Rectifiers. New York: McGraw-Hill, 1948.

[16] G. C. Messenger and C. T. McCoy, "Theory and operation of crystal diodes as mixers," Proc. IRE, vol. 45, pp. 1269-1283, Sept. 1957.

[17] S.T.Eng, "Low-noise properties of microwave backward diodes," IRE Trans. Microwave Theory Tech., vol. MTT-9, pp. 419-425, 1961.

\title{
Automatic Test System for an Analog Computer
}

\author{
J. FRANK ROSS, DOUGLAS G. PINCOCK, MEMBER, IEEE, ROBERT J. KAVANAGH, AND DAVID M. WANAMAKER
}

\begin{abstract}
This paper describes the design, construction, and performance of an automatic test system for the analog portion of a hybrid computation system. The system, which is capable of carrying out complete dynamic testing of each analog component, uses the digital portion of the hybrid system to provide the desired control with the result that the requirement for sophisticated stimulation and measurement equipment is minimized. The hardware and software of the system are both modular in nature in order to provide versatility and to facilitate modifications and additions as required. The design philosophy is such that the methods used are not limited to a particular analog computer. In fact, they have applications in the testing of many types of electronic equipment.
\end{abstract}

\section{INTRODUCTION}

$\mathrm{O}$ NE OF THE MAIN difficulties in making efficient use of hybrid computation systems is the fact that failures in the analog subsystem are relatively frequent. It is, of course, important that such failures be isolated before they introduce errors into solutions. This can be accomplished to a certain extent through extensive use of static checks by programmers [1], [2]. These checks, however, provide no guarantee that

Manuscript received April 30, 1973; revised July 18, 1973. This work was supported by the National Research Council of Canada under Grant A5155. This paper was presented at the 1973 Electrical and Electronic Measurement and Test Instrument Conference (EEMTIC), Ottawa, Ont., Canada, May 15-17.

J. F. Ross is with the Defense Research Establishment Valcartier, Defense Research Board, Valcartier, P.Q., Canada.

D. G. Pincock, R. J. Kavanagh, and D. M. Wanamaker are with the Electrical Engineering Department, University of New Brunswick, Fredericton, N.B., Canada. components are operative in a dynamic sense, i.e., that integrators do in fact integrate, that nonlinear function generators work in all quadrants, that the frequency response of amplifiers is adequate, etc. Therefore, if users are to have confidence in a hybrid system, it is essential that frequent preventative maintenance tests be carried out on all components. Unfortunately, such tests tend to be very time consuming and hence, are carried out relatively infrequently. Due to the presence of a general-purpose digital computer in the hybrid configuration, it seems natural to investigate the use of automatic testing as a solution to this problem. This paper describes the development of an automatic test system for the analog portion of the EAI 590 Hybrid Computation system at the University of New Brunswick, Fredericton, N.B., Canada.

One of the main considerations in the design of the automatic test system was to minimize costs by using the digital computer as much as possible in place of sophisticated stimulation and measurement equipment. In addition, both hardware and software were designed to be modular in nature in order to provide versatility and facilitate modifications and additions as required.

\section{Overview of the Test System}

Stuehler [3] divides any general automatic test system into the following logical units, as shown in Fig. 1:

1) a unit which is under test (UUT); 